\title{
Lambert's Cosine Law and Sidescan Sonar Modeling
}

\author{
John Folkesson ${ }^{1}$, Hongsheng Chang ${ }^{1}$, Nils Bore ${ }^{1}$
}

\begin{abstract}
When a planar surface area reflects diffusely, the reflected rays have a distribution of directions as opposed to specular reflection which is in a single direction. Ideal diffuse reflection, aka Lambertian reflection, has equal reflected energy at all angles away from the plane. This leads to Lambert's cosine law that says the observed radiance from the area will be proportional to the cosine of the incidence angle. This result has been interpreted in differing ways which we will address here to show that the same reasoning leads to other 'laws' in other situations. The key is that Lambert's law refers to a defined illuminated area of a plane and the energy coming off that area, for an ideal diffuse reflector when irradiated at a particular angle of incidence. It is the result of the geometry that the range of incident angles of light reaching the observer will follow exactly the cosine law. In the case sidescan sonar the area being ensonified also changes with the angle as does the area contributing to the reflected energy in a particular time bin. This changes the cosine law to a cotangent law. The sine in the denominator is due to the changing size of the area being observed at different angles.
\end{abstract}

\section{INTRODUCTION}

Sidescan sonar is appealing as a workhorse sensor for seabed surveying due to its simple design, relative low cost, long range and ease of hull mounting. The images produced are also of fairly good resolution compared to other sonars. The drawback is that they do not give the full geometry of the seabed but rather a $2 \mathrm{D}$ projection of the $3 \mathrm{D}$ surface. It is therefore of interest to see if the images produced can be modeled in terms of the surface normal directions and thereby gaining some understanding of the third dimension from intensity variations.

Sound is a wave and behaves as light does when reflected by a surface. Therefore there has been work done to transfer the shape from shading work from camera images to sidescan images. One must be careful to take account of the subtle differences in the way the sidescan sensor forms its signal. Cameras provide an intensity within a fixed small solid angle. Sidescan has a fixed angle in one dimension but the 'pixel' size in the other is set by a range interval, not an angle.

According to [1], sidescan has three types of sidescan backscatter reflection processes, facet, mixed and microscale roughness backscatter. These correspond to angles of incidence near 0 , middle range or grazing respectively. Alternatively, the backscatter can be split into a specular and diffuse reflection. It is the diffuse reflection that contains the most information. Contrary to popular belief, diffuse reflection has nothing to do with roughness of the surface

\footnotetext{
*This work was supported by Stiftelsen för Strategisk Forskning (SSF) through the Swedish Maritime Robotics Centre (SMaRC) (IRC15-0046).

${ }^{1}$ Robotics, Perception and Learning Division at KTH Stockholm, Sweden \{johnf, changh, nbore\}akth.se
}

but rather it is energy that is absorbed and re-emitted in all directions. However, irregularities in the surface smoothness does effectively increase other types of reflection that may mask the diffuse component.

For an 'ideal diffuse reflector' backscatter strength will be uniform in all directions from the reflecting surface. That is, when changing the direction of the receiving transducer and keeping the sound source location fixed, the energy reflected into a given solid angle from the surface is the same in all directions. There will be however a change in strength depending on the direction of the ensonification. This is what allows the surface geometry to be partially inferred from the sidescan image.

Specular reflections are, for the most part problematic for estimating contours from sidescan. Having the source and receiver at the same position means that these reflections occur near incidence angles of 0 . For seabed surveys this mostly occurs close to the nadir although steep slopes from rocks or holes can produce them further out from the track.

Our main contribution here is to resolve the confusion over what diffuse reflection implies as to surface normal for sidescan sonar. In the literature two models have been used. One uses the $\cos$ of the incident angle and one the $\cos ^{2}$. We show first analytically and then by careful measurements at sea that the relation is in fact cot.

We present first our model of sidescan sonar from an ideal diffuse reflective planar surface. Then we discuss why this model differs from some other models that have been tried.

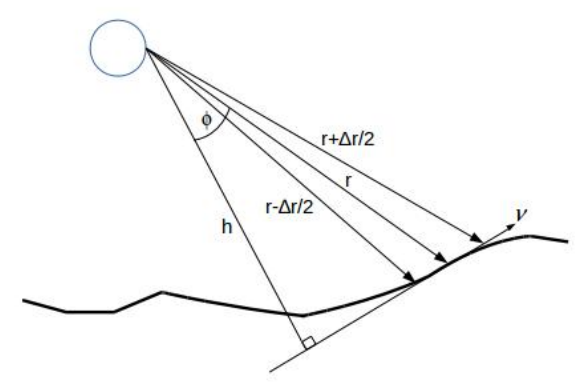

Fig. 1: A schematic sketch of the sidescan beam geometry.

\section{SIDESCAN SONAR AND THE COTANGENT LAW}

\section{A. Problem description and limitations}

Sidescan sonar has a beam pattern that is very wide in the vertical direction (sometimes called the azimuth direction) and very narrow in the longitudinal, i.e. direction parallel to the linear transducer array. The received signal comprises backscatter intensities received over intervals of time after 
the ping was sent. The time interval depends on the effective pulse width of the sonar transmitter [1]. These are arranged in a vector of time bins. Each bin has the received reflected intensity over a range of distances from the sonar array from regions of the seafloor. In general there can be multiple reflections but for forming the models here we will always assume there is one planar surface creating the received reflected signal for a bin.

A further consideration is the time varying gain, TVG, that must be applied to the sonar signal to compensate for the attenuation in the water volume. This multiplies the signal by some, for us, arbitrary function of $r$. Absorption in water is normally modeled as being exponential with the range. Such an exponential correction is normally applied in post processing as a standard image enhancement method. The sonar itself often applies some default TVG to the signals before storing them, in order to limit the dynamic range and thus the size of the data, but as that value is hard to know a priori a further exponential correction can help.

In [2] they show an example of both the 'residual TVG' and an estimate of the sidescan beam pattern. The beam pattern has several lobes. Their estimate was based on [3]. We will discuss this further in the related work section. In our case we have a beam pattern for the sonar from the manufacture that we can use. For a linear transducer array the beam pattern can be computed analytically. This idealized model predicts that for our sonar we will have only the main lobe contribute to our data.

The problem can be analyzed in 2 dimensions, vertical and transverse. As we said, the beam is very narrow in the longitudinal direction (i.e. direction of travel in survey applications) and we will be able to add in this third dimension at the end. The reason this will work is that the angle in the longitudinal direction subtended by the area of plane reflected into a particular bin of sidescan is not a function of the range or plane normal. The area of the sea floor is bigger further away but the energy hitting it is the same, fig. (2). For this reason the inclination of the plane in the longitudinal direction will not matter (and can not be measured). This will become clearer when we discuss the narrow beam sonar and show that the signal in the case of fixed angluar beam width does not vary with the normal direction (in this theoretical analysis).

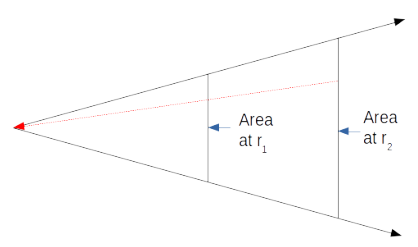

Fig. 2: The energy incident and diffusely re-radiated in an angular aperture does not depend on range, $r$.

Sound is refracted by the changing sound velocity profile, SVP, in the water but this effect will not be considered here to keep the discussion simple. So we assume no absorption and a uniform SVP for the analysis of the problem. When we actually take measurements these factors are part of the post processing.

There would also be a factor less than one to account for the proportion of the signal that is actually reflected diffusely by the seabed. Mud for example can reflect very faintly [4]. We are not including that multiplicative factor here either. Our formula is mainly to find the dependency between backscatter and incidence angle.

\section{B. Analysis}

We see from fig. (1) that the perpendicular distance, $h$, between the sonar and the reflecting plane is:

$$
h=r \cos (\phi)
$$

and by taking the derivative:

$$
d \phi=\frac{d r}{r} \cot (\phi)=h \frac{d r}{r \sqrt{r^{2}-h^{2}}} .
$$

Where $r$ is the distance traveled from the sonar to the point on the plane. For simplicity we will call $\phi$ the 'incidence' angle although it is actually the angle of the projection to the 2D transverse plane. As we have said, the part of the normal in the longitudnal direction does not actually influence the signal.

The signal received in a particular time bin centered on distance $r$ will arise from reflections traveling the distances between $r-\frac{\Delta r}{2}$ and $r+\frac{\Delta r}{2}$. These two endpoints for the distance range correspond to endpoints in a range of incident angles for the ensonifying beam of $\phi-\Delta \phi_{1}$ and $\phi+\Delta \phi_{2}$.

We can thus compute the range of angles in the bin:

$$
\begin{aligned}
\Delta \phi & =\Delta \phi_{1}+\Delta \phi_{2} \\
& =\int_{-\Delta \phi_{1}}^{\Delta \phi_{2}} d \phi \\
& =\int_{r-\Delta r / 2}^{r+\Delta r / 2} h \frac{d r}{r \sqrt{r^{2}-h^{2}}} \\
& =\left.\arccos \frac{h}{r}\right|_{r-\Delta r / 2} ^{r+\Delta r / 2} \\
& \approx \frac{\Delta r}{r} \cot (\phi)
\end{aligned}
$$

Here the approximation uses the fact that $\Delta \phi<<1$ and that $\Delta r<<r$. Also $r-\frac{\Delta r}{r}>h$ must hold. Thus we find that the ensonification process has a range of incidence angles over the range 'bin' area of $\frac{\Delta r}{r} \cot (\phi)$.

We assume that the energy emitted by the sonar array will be spread uniformly over a wide range of angles including this small interval. The energy hitting this small planar area is thus proportional to the range of incident angles.

$$
E_{r} \propto \frac{\Delta r}{r} \cot (\phi) .
$$

This energy will be reflected according to the ideal diffuse reflection in all angles uniformly. If the transducer 2-way 
beam pattern is constant over this angular interval then the intensity of the received signal will be:

$$
S_{r} \propto \frac{\Delta r}{r^{3}} \cot (\phi)
$$

Where the additional $r^{2}$ term comes from beam spreading of the return signal.

If this analysis does not convince there is a second way to obtain this result. If the beam width in the longitudinal direction is $\alpha<<1$, then the corresponding side of the rectangular region of the plane corresponding to the bin projected to the array direction $x$ is

$$
\Delta x=\alpha r
$$

and in the transverse direction it is (see page 31 of [1]):

$$
\Delta v \approx \frac{\Delta r}{\sin \phi} .
$$

We use $v$ to signify the direction along the plane in our two dimensional fig. (1). We emphasise that $x$ is not along the plane and would be horizontal if the array is horizontal. The incident energy per unit projected area and solid angle of uniform reflection is

$$
I_{r} \propto \frac{\cos (\phi)}{2 \pi \alpha r^{2}} .
$$

The same formula holds in the other cases we shall look at and is in fact Lambert's law in 2D. But we still need to account for the observer process.

If we assume that the sonar has an effective receive 'area' of $A_{s}$ then the energy reflected in a solid angle

$$
\frac{A_{s}}{r^{2}}
$$

will be detected. We thus can compute the energy that reaches the bin, $S_{r}$, by multiplying $I_{r}$ by the area $\Delta x \Delta v$ and by this solid angle.

$$
S_{r} \propto \frac{A_{s}}{r^{2}} I_{r} \Delta x \Delta v \propto \frac{A_{s}}{r^{2}} \frac{\Delta r}{2 \pi r} \cot (\phi) \propto \frac{\Delta r}{r^{3}} \cot (\phi)
$$

The power of 3 might be surprising but it comes from the fact that the incident beam is modeled as covering an area in the along track $x$ direction proportional to $r$ which is all then reflected uniformly and thus no energy is lost due to spreading in the $x$ direction for the incident ray. We notice that had we computed the factor to compute the actual plane side length in eq. (5), it would have cancel just as $\alpha$ does in eq. (9). Interestingly while the $\cot (\phi)$ and $\cos (\phi)$ behave very differently, they are rather similar for values of $\phi$ close to $\frac{\pi}{2}$. Those shallow grazing values might be found in the farthest ranges in the scan. As the angles get smaller the $\cos ^{2}(\phi)$ is a better fit for the relative slope of the $\cot (\phi)$ curve, matching it exactly at $\pi / 4$, fig. (3). This might explain why some find the square of the cos to be empirically better at modeling the sidescan. Ultimately for smaller angles the rate of change of the signal becomes very large.

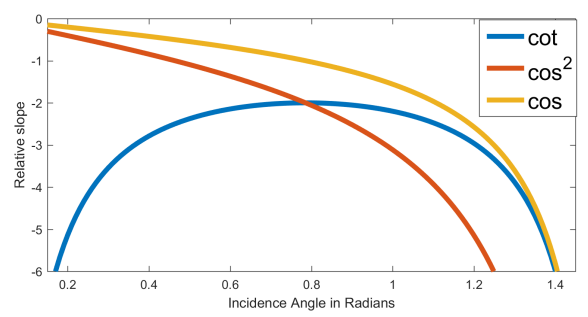

Fig. 3: Here we plot the relative slope, $f^{\prime}(\phi) / f(\phi)$ for three functions. Relative slope allows inferring bottom shape.

\section{LAMBERT'S COSINE LAW}

The situation usually envisioned in Lambert's cosine law with the observation is as if there is an area of diffuse reflection surrounded by non-reflective regions. This could be a small plane suspended in space for example. The result is very similar to in the previous section until we compute the received signal. Now the $\Delta v$ will not have any $\phi$ dependence and $\Delta x$ will not have any $r$ dependence. So we would simply get a signal obeying a $r^{-4} \cos (\phi)$ law with $\phi$ now the true $3 \mathrm{D}$ angle of incidence. This is Lambert's cos law and is consistent with the previous result if we consider that in that situation we have a range interval for the observation that causes $\Delta v$ to vary like $\sin ^{-1}(\phi)$. And the $\Delta x$ is proportional to $\mathrm{r}$,

\section{NARROW Multibeam SONAR}

In this section we consider a subset of multibeam sonar which have a narrow beam width in both directions. The sonar has two arrays at right angles to one another. One does the ensonification similar to a sidescan array while the other listens to separate angle channels each of which can have time bins as in sidescan but here the bins give so called water column intensities. Often the return from the sea bed is extracted as a single return for the channel as a distance. This gives a $3 \mathrm{D}$ point of reflection.

For the ensonification part one can imagine breaking the surface up into small fixed areas. We see that the ensonification intensity per unit area will be as before with an $r^{-2} \cos (\phi)$ dependence.

However now we are in a third situation in which $\Delta \phi$ is fixed rather than $\Delta r$ as in sidescan sonar or $\Delta v$ in Lambert's cosine law. Thus we have

$$
\Delta v=\frac{r \Delta \phi}{\cos (\phi)} .
$$

This $\Delta \phi$ is not changing with $\phi$ as it was for the sidescan. We thus get a signal at this angle channel, $\theta$, of

$$
\begin{aligned}
S_{\theta} & \propto \frac{\Delta s}{r^{2}} \frac{\Delta \phi}{2 \pi} \\
& \propto \frac{1}{r^{2}}
\end{aligned}
$$

The conclusion is that for 'narrow' multibeam sonar the backscatter intensity should not depend on the normal 
direction relative to the beam, see fig.(2). The surface area within a solid angle changes with $\phi$ but the energy hitting it will not. Note the difference from the camera case, which is also a fixed angular aperture, is that for a camera the illumination (which defines the angle of incidence) is from a separate source.

Note that the conclusion of no dependence assumes that there is no range bin used in the sonar to produce the returned intensity. All ranges that hit the bottom within the solid angle would be included in the return intensity. In fact, the signal will be processed over range bins and the return from the bottom may be a sum of the bins associated with the bottom or simply the highest bin return value. The later would give something more complicated than either of the cases analysed here.

\section{FORWARD LOOKING SONARS, FLS}

So called forward looking sonars are also called multibeam sonars. Some of these are implemented by mechanically scanning. However each of the beams are similar to the sidescan beams in that one direction has a relatively wide beam and the signals for each beam are received as a vector of time bins as with the sidescan. When the wide beam is oriented vertically and directed so as to graze the seabed surface, the situation is exactly as in the sidescan and the same law for received energy per bin applies.

\section{RELATED WORK}

In [5], [6], [7] sidescan sonar is modeled as following a $\cos (\phi)$ law. This is of course a rather natural choice at first based on Lambert's law, eq. (7), as described for sidescan sonar in [8]. We have, however, shown that this may not be the best model based on modeling the change in size of the area of reflection with incidence angle.

One reason that cos law was first applied to sonar is that the early works were influenced by shape from shading works done for camera images. Cameras are sensors that have narrow angular 'beams' and do not themselves send out light to be reflected. This makes them quite different to sidescan sonar and it should be clear that for bins (pixels) that represent small fixed angular ranges observing surfaces illuminated from a third position the cos law does apply.

There is some work on modeling mechanical scanning and FLS. In [9], [10] they model the received energy as proportional to $\cos ^{2}(\phi)$. [10] cites [11] as the source of what they call 'Lambert's reflection law'. [9] cites [12] which states that 'Lambert's rule' is an empirical rule that gives the $\cos ^{2}(\phi)$ dependence. The mechanically scanned sonar used in that work has a beam pattern similar to a sidescan for each mechanically scanned angle. We have ourselves observed that often a power of 2 helps model the sidescan better than the cos law does. This is often attributed to the ideal diffuse reflection not actually holding but it may be that the cot should be used instead.

In [13], [3] they carefully estimate both the residual TVG and the angular beam pattern of the sidescan sonar. As the angular beam pattern, in fig. 4 of [3], gets closer to normal incidence it levels off and then exhibits a second peak due to the side lobe pattern of the sidescan. They also show that this side-lobe effect causes the response to drop to 0 rapidly from about 70 to 90 degrees from the center of the beam. One can see that their sidescan is only usable for inferring normals in the middle section. Elsewhere the curve flattens or becomes distorted by the side-lobe effects making it useless for that purpose.

The way that they measure the beam pattern is to assume a flat bottom and have some variation in altitude. Under this assumption one can not separate the beam pattern from the incidence angle so that on can not fit those curves to our model. However in fig. (5) of [13] which is copied from [14] One can see that the curves for backscatter into a mechanically scanned sonar tend to look more and more like the tangent of the grazing angle which is to say the $\cot (\phi)$ as one moves from rough rock to fine silt seabed types. This seems to support our analysis. The rough rock can be considered to be dominated by specular reflections from rock faces aligned with $\phi$ whereas one would expect that that effect would disappear for fine silt as the faces would be so much smaller than the wavelength..

\section{EXPERIMENTAL METHOD}

We have collected data using a Kongsberg Hugin AUV equipped with EM2040 Multibeam and Edgetech 2005 sidescan sonars. We surveyed an area of a fjord with a relatively flat but varyingly sloped seabed consisting of very fine slit at two different altitudes. This should give a diffuse reflection for the shallower angles.

We form a mesh out of the multibeam data. This mesh is used to determine for each intensity return from the sidescan, the altitude, range, beam angle and incidence angle.

The sidescan waterfall image has a row for each ping and each row has columns for the various range bins. So the $i^{t h}$ bin is associated with some range, $r_{i}$. For each bin there is a signal intensity, $S_{i}$. We will be assuming each of these is from it's own planar surface with center coordinates in the horizontal plane of $\left(x_{i}, y_{i}, z_{i}\right)$. There are two sonars, port and starboard, (left and right). Each with its own position $\left(x_{p}, y_{p}, z_{p}\right)$ and $\left(x_{s}, y_{s}, z_{s}\right)$. We found that there is no significant difference in the two sonars so the data was combined. We associate 4 numbers to each data point:

1) Intensity, $S_{i}$;

2) 'Altitude', $a_{i}$, the difference in vertical depth passed by the ray, for example for the port sonar $a_{i}=z_{p}-z_{i}$;

3) the beam angle $\theta=\operatorname{asin}\left(a_{i}, r_{i}\right)$;

4) the incidence angle, $\phi$.

For a flat horizontal seabed $\theta+\phi=90^{\circ}$ and $\theta, \phi$ and $r$ are strongly correlated in the data. This will require us to use models for the beam angle and range correction in order to separate out the incident angle correction. We start by accumulating the data into intervals for the four dimensions $(a, r, \theta, \phi)$. For the beam angle we use a correction of

$$
c(\theta)=\left(\frac{k \sin \left(\theta-\theta_{0}\right)}{\sin \left(k \sin \left(\theta-\theta_{0}\right)\right)}\right)^{4}
$$


with $k=2.78$ for a (one-way) $3 \mathrm{~dB}$ beam width of $60^{\circ}$. This is based on the theoretical beam pattern of a linear array for a large number of elements. The ET2005 sonar specifies this beam width. Kongsberg has mounted the sonar with the recommended angles of depression, $\theta_{0}=30^{\circ}$. With these values the sidelobes do not appear in our data.

We must remove outliers from shadows, (dark regions) and objects/rocks, (bright pixels) from our data. We also must estimate the residual TVG for this environment. Additionally we will compute a correction for altitude to mainly allow for differences in the water layers when we repeat the survey at higher altitudes. We started by modelling the range correction as a $6^{\text {th }}$ degree polynomial. The altitude correction was estimated as 6 discrete correction factors for $11,16,21,25$, 28 , and 31 meters altitudes. The $\phi$ correction was estimated as discrete correction factors for .03 radian intervals and interpolated to .01 radian intervals.

We fit the correction factors by minimizing a loss function

$$
L=\frac{1}{2} \sum_{a, r, \theta, \phi}\left(C(a)(C(r) C(\theta) C(\phi) I(a, r, \theta, \phi)-\bar{I})^{2}\right.
$$

where the $C$ are the correction factors/functions. With these assumptions, the minimization can be done exactly when taken one dimension at a time, i.e only the $C(a)$ holding the others fixed and so on. We could also do a gradient descent on several factors at once. Due to the strong correlations between the factors, the order of doing this did matter. For example, if we do not fit $C(\phi)$ first we end up 'explaining' most of the variations with the other factors, significantly flattening the $C(\phi)$ curve. We found that estimating in order $C(\phi), C(r), C(\phi),(C(r) \& C(\phi)), C(r), C(\phi), C(a), C(\phi)$, $(C(a) \& C(\phi))$, gave a good balance between the factors. We started with $\mathrm{C}(\mathrm{r})=1$ an $\mathrm{C}(\mathrm{a})=1$. We first use all the data with no outlier removal. After optimization of the corrections, we removed the brightest $5 \%$ of the outliers above the mean and darkest $5 \%$ below the mean after applying the corrections. We then repeated the fitting process.

While this did produce $C(\phi)$ curves that were reasonable, there was still evidence of over fitting. We suspected that a too general range model drove the solutions to physically unreasonable values. Specifically, the $6^{\text {th }}$ degree polynomial for $C(r)$ gave an $S$ shaped curve that did not seem to be reasonable. We therefore decided to also use a more conventional exponential attenuation with $0.045 \mathrm{~dB}$ per $\mathrm{m}$ attenuation. That attenuation value matched the range of the $C(r)$ values and the overall trend from the polynomial fit. According to the formula in [15] using the temperature salinity and temperature for our environment the total acoustic 2-way attenuation should be about $0.200 \mathrm{~dB}$ per $\mathrm{m}^{1}$, which does not include beam spreading effects. Using the graph in the Edgetech sidescan application note ${ }^{2}$ we find that their loss in seawater curve fits well to a forth order $r$ term

\footnotetext{
${ }^{1}$ http: //resource.npl.co.uk/acoustics/techguides / seaabsorption/.

${ }^{2}$ https: //www. edgetech.com/ edgetec3/wp-content/ uploads/2019/07/app\_note\_range.pdf
}

times an exponential term of between .31 and $.37 \mathrm{~dB}$ per $\mathrm{m}$. These attenuation values are also consistent with [15] (.34 $\mathrm{dB} / \mathrm{m}$ ) at the higher water temperature for that data. We are multiplying our residual correction to the unknown TVG of the sonar. We repeated the above fitting and outlier removal procedures but did not fit $\mathrm{C}(\mathrm{r})$ further.

\section{RESULTS}

The result when using the $6^{\text {th }}$ degree polynomial for $C(r)$ are shown in fig. (4). Here we fit the three functional forms over the interval 0.03 to 1.47 of incident angles. In fig. (5) we show the mean square error, MSE, between the curves fit over other intervals. The plot is logarithmic.

The result when using $0.045 \mathrm{~dB}$ per m residual attenuation for $C(r)$ are shown in fig. (6). Here we again fit the three functional forms over the interval 0.03 to 1.47 of incident angles. In fig. (7) we again show the mean square error between the curves fit over other intervals.

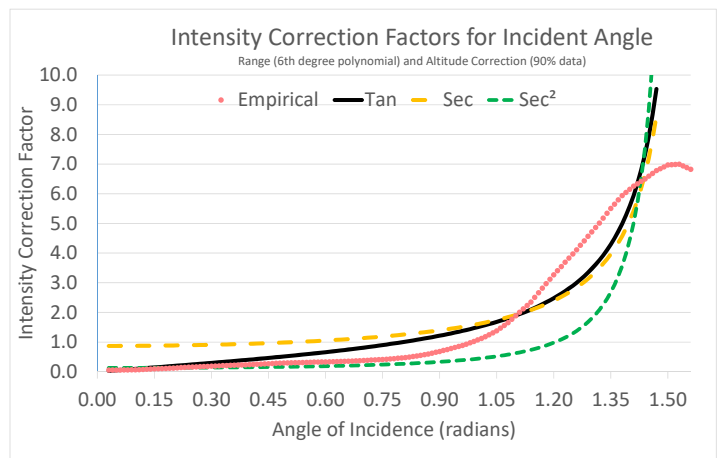

Fig. 4: The three curves were fit to the empirical values over the interval 0.03 to $1.47 \mathrm{rads}\left(C(r) 6^{\text {th }}\right.$ degree polynomial).

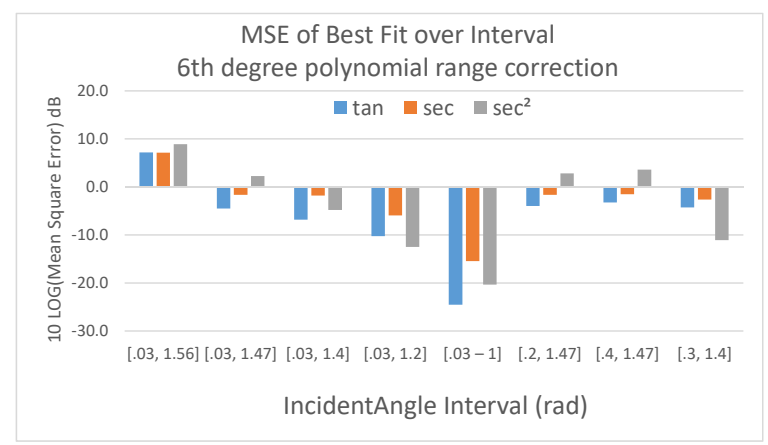

Fig. 5: Comparison of the fit to the correction factor for the three curves over various intervals when the polynomial correction was used for $C(r)$. 


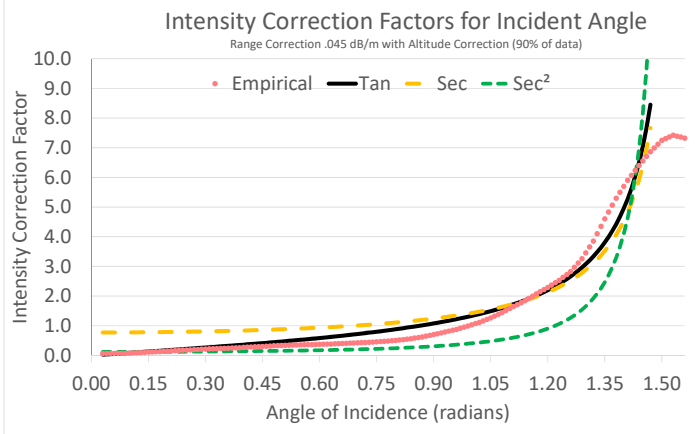

Fig. 6: The three curves were fit to the empirical values over the interval 0.03 to $1.47 \mathrm{rads}$ (exponential $\mathrm{C}(\mathrm{r})$ ).

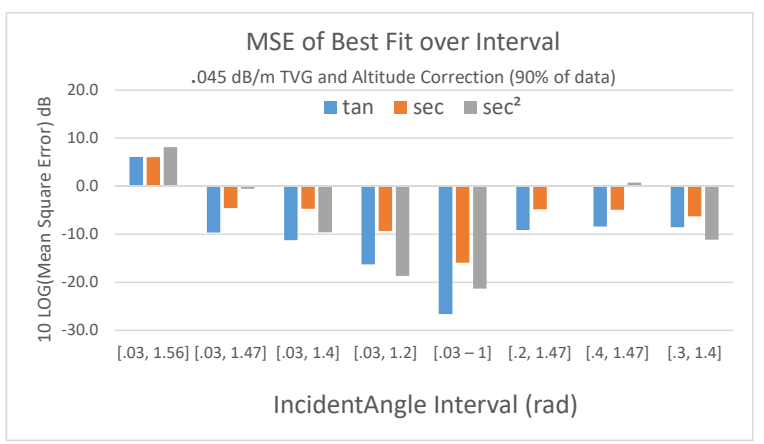

Fig. 7: Comparison of the fit to the correction factor for the three curves over various intervals when $0.045 \mathrm{~dB}$ per $\mathrm{m}$ residual attenuation correction was used for range.

\section{Discussion}

We see in fig. (4) and fig. (6) that the correction factor curves, which are the reciprocal of the intensity dependence, do indeed follow a general $\tan (\phi)$ shape. At the higher angles the empirical data begins to show a tendency toward constant or even decreasing corrections. This is most likely due to the fact that these shallow grazing angles often come from the protruding rock formations near the edges of the fjord. Either that or some other material properties of the bottom are often different for these angles. Surface reflections are also know to add noise to sidescan at the higher angles. It can be noticed that the effect is greater for the polynomial model which may indicate we were better able to remove those outliers with the exponential model.

Looking at fig. (7) we see that the fit for the tan was significantly better for most intervals. For $\phi \in(0.03,1.47)$ the tan was clearly best. When including the previously mentioned questionable data from 1.47 to 1.56 rads the three models seemed about equally as bad. When we only consider smaller interval of the $\phi$ range we see that $\tan$ and $\sec ^{2}$ are in some nearly the same. This agrees with our observation that in the mid range the relative slopes of these two curves are similar, fig. ((3).

It can be seen that the sec curves come closest to the tan curves in terms of MSE fit when the interval includes the larger angles. This agrees with our observations and fig. ((3).

\section{Conclusion}

Our analysis of the sidescan sonar for diffuse reflection shows a dependence on angle of incidence of $\cot (\phi)$. We have confirmed that this does indeed hold for data collected from a silt bottom using an Edgetech 2005 sidescan sonar along with a mesh built from multibeam sonar data.

\section{ACKNOWLEDGMENT}

This work was supported by Stiftelsen for Strategisk Forskning (SSF) through the Swedish Maritime Robotics Centre (SMaRC) (IRC15-0046). We would also like to thank MMT and Prof. Anna Wahlin of Gothenburg University for helping us to collect the data used for this article.

\section{REFERENCES}

[1] P. Blondel, The Handbook of Sidescan Sonar. Springer, 2009.

[2] S. Reed, I. T. Ruiz, C. Capus, and Y. Petillot, "The fusion of large scale classified side-scan sonar image mosaics," IEEE Transactions on Image Processing, vol. 15, pp. 2049-2060, July 2006.

[3] C. Capus, I. T. Ruiz, and Y. Petillot, "Compensation for changing beam pattern and residual tvg effects with sonar altitude variation for sidescan mosaicing and classification," in Seventh European Confelanrence on Underwater Acoustics, ECUA, July 2004.

[4] D. P. Williams and J. Groen, "A fast physics-based, environmentally adaptive underwater object detection algorithm," in OCEANS 2011 IEEE - Spain, pp. 1-7, June 2011.

[5] E. Coirasa and J. Groena, " $3 \mathrm{~d}$ target shape from sas images based on a deformable mesh," 2009.

[6] E. Coiras and J. Groen, "Simulation and 3d reconstruction of sidelooking sonar images," 2009.

[7] E. Coiras, Y. Petillot, and D. M. Lane, "Multiresolution 3-d reconstruction from side-scan sonar images," IEEE Transactions on Image Processing, vol. 16, pp. 382-390, Feb 2007.

[8] D. Langer and M. Hebert, "Building qualitative elevation maps from side scan sonar data for autonomous underwater navigation," in Proceedings. 1991 IEEE International Conference on Robotics and Automation, pp. 2478-2483 vol.3, April 1991.

[9] E. I. Thorsos, K. L. Williams, N. P. Chotiros, J. T. Christoff, K. W. Commander, C. F. Greenlaw, D. V. Holliday, D. R. Jackson, J. L. Lopes, D. E. McGehee, J. E. Piper, M. D. Richardson, and Dajun Tang, "An overview of sax99: acoustic measurements," IEEE Journal of Oceanic Engineering, vol. 26, pp. 4-25, Jan 2001.

[10] M. D. Aykin and S. Negahdaripour, "Forward-look 2-d sonar image formation and 3-d reconstruction," in 2013 OCEANS - San Diego, pp. 1-10, Sep. 2013.

[11] R. Urick, Principles of underwater sound. McGraw-Hill, 1983.

[12] D. R. Jackson, K. L. Williams, and K. B. Briggs, "High-frequency acoustic observations of benthic spatial and temporal variability," GeoMarine Letters, vol. 16, pp. 212-218, Sep 1996.

[13] C. Capus, A. Banks, E. Coiras, I. T. Ruiz, C. Smith, and Y. Petillot, "Data correction for visualisation and classification of sidescan sonar imagery," IET Radar, Sonar and Navigation, vol. 2, pp. 155-169(14), June 2008.

[14] D. R. Jackson, "Apl-uw high-frequency ocean environmental acoustic model handbook," tech. rep., APL-UW, 1994.

[15] M. A. Ainslie and J. G. McColm, "A simplified formula for viscous and chemical absorption in sea water," The Journal of the Acoustical Society of America, vol. 103, no. 3, pp. 1671-1672, 1998. 